\title{
Temporal and parietal association cortex lesions and spatial and black-white reversal learning in the rat
}

\author{
WILLIAM F. MCDANIEL and ROGER K. THOMAS \\ University of Georgia, Athens, Georgia 30601
}

\begin{abstract}
In Experiment 1, rats with parietal, temporal, or frontopolar neocortex ablations and nonoperated controls were trained on seven spatial reversals. The animals with parietal association cortex damage were found to be deficient on this task compared to all other groups. In Experiment 2, rats with either temporal or striate neocortical ablations or subcortical lesions of the lateroposterior thalamic nucleus and nonoperated controls were trained on a black-white reversal task. The rats with temporal damage committed significantly more errors than all other groups. These results together with those of previous research suggest that a dissociation exists between the effects of parietal and temporal association cortex ablations on reversal learning in the rat. It was suggested that the visual functions of the temporal association cortex might be mediated by connections with either the striate cortex or the lateroposterior thalamic nucleus.
\end{abstract}

Several studies investigating the anatomy (e.g., Lashley, 1941; McDaniel, McDaniel, \& Thomas, 1978) and behavioral functions (e.g., Boyd \& Thomas, 1977; Thomas \& Weir, 1975) of the posterior association cortex of the rat have suggested that there are two dissociable subdivisions within this region. Lashley (1941) distinguished between these regions anatomically by investigating the primary sources of thalamic afferents innervating them. The parietal cortex, located rostral to the occipital cortex, receives projections from the lateral thalamic nucleus. A second region, lying rostrolateral to the striate cortex and corresponding mostly to Krieg's (1946) area $18 \mathrm{~A}$, was found to receive thalamic projections from the lateroposterior nucleus. McDaniel et al., (1978) replicated Lashley's anatomical findings concerning these two regions using both retrograde degeneration and horseradish peroxidase techniques. These authors suggested that the temporal cortex in the rat may be a region homologous to the temporal association cortex of the tree shrew, which is considered by some to represent forms ancestral to primates (Diamond \& Hall, 1969; Harting, Hall, \& Diamond, 1972; Killackey, Snyder, \& Diamond, 1971). Hughs (1977) has also investigated the projections of nucleus lateroposterior using autoradiography and horseradish peroxidase techniques and has shown that this nucleus contributes efferent projections to areas 18A, 18, 7, and 20 (Krieg, 1946).

Lesions of the temporal cortex in the rat are associated with deficiences in black-white reversal

Reprint requests should be addressed to William F. McDaniel, Department of Psychology, Georgia College, Milledgeville, Georgia 31061. learning and deficits on learning both simple and complex visual pattern discriminations (Boyd \& Thomas, 1977). Temporal lesions did not, however, disrupt performance on the Hebb-Williams mazes. Lesions of the parietal cortex in the rat have been consistently associated with learning deficits on mazes and maze-like tasks (Ain, Lubar, Moon, \& Kulig, 1969; Boyd \& Thomas, 1977; Forgays, 1952; Gross, Chorover, \& Cohen, 1965; Krechevsky, 1935; Lansdell, 1953; Thomas, 1966; Thomas \& Weir, 1975) and deficits on a complex visual pattern discrimination. Parietal lesions did not disrupt simple pattern discrimination or black-white reversal learning.

To further examine the nature of the deficits which accompany lesions in the parietal and temporal cortices, two experiments were conducted with rats that had received either experimental or control lesions. In Experiment 1, a spatial reversal task was used to examine the effects of parietal lesions on spatial problems and to examine the generality of the temporal deficits on the reversal problems reported by Boyd and Thomas (1977). In Experiment 2, the importance of the integrity of the connections from the lateroposterior thalamic nucleus to the temporal cortex in performance on a black-white reversal task was examined.

\section{GENERAL METHODS}

\section{Subjects \\ Forty-eight male and female hooded rats (Long-Evans strain bred at the University of Georgia), ranging in age from 90 to 120 days at the time of surgery, were used. The animals were housed individually and maintained on a light-dark cycle with light onset at 8:00 p.m. and offset at 8:00 a.m. local time. All}


testing was done during the dark phase. In both experiments, the rats were randomly assigned to the experimental groups, with the restriction that each group have equal numbers of males and females.

\section{Surgery and Histology}

All surgery was performed with the rat anesthetized with Equithesin $(0.30 \mathrm{ml} / 100 \mathrm{~g}$ body weight). Occasionally, ether was used as a supplemental anesthesia. Clean surgical techniques were used. After a midline incision was made and the dorsal cranium exposed, depending upon the lesion, the skull was drilled with a trephine at the coordinates indicated in Table 1 . The underlying cortical matter was aspirated. An attempt was made to place the temporal and parietal ablations within the boundaries of Lashley's (1941) designated target areas for projections from the lateroposterior thalamic nucleus [i.e., temporal cortex; see Lashley's (1941) Figures 17 and 18, p. 85] and for projections from the lateral thalamic nucleus (i.e., parietal cortex; see Lashley's Figure 15, p. 85). The lateroposterior lesions used in Experiment 2 were made with a Grass lesion maker (LM-3). Upon completion of surgery, the scalp was sutured and Amipher topical antiseptic dressing was applied to the wound. The animals were kept warm until recovery from anesthesia was complete.

At the end of each experiment, the animals were anesthetized and perfused intracardially with $50 \mathrm{cc}$ of $0.85 \%$ saline followed by $50 \mathrm{cc}$ of $10 \%$ Formalin. The brains were stored in Formalin for a minimum of 7 days before being photographed from the dorsal view. The photographs were used to determine the percentages of ablated cortex, determined by the dot-grid method (Thomas \& Peacock, 1965), and the boundaries of the region destroyed. The brains were then embedded in celloidin, sectioned at $40 \mu \mathrm{m}$, mounted on slides, and stained with cresyl violet.

\section{EXPERIMENT 1}

As noted earlier, several investigators have reported deficits in rats with parietal lesions on mazes and maze-like tasks. Such deficits may suggest a role for the parietal cortex in spatial behavior, but deficits were not seen on a spatial reversal task in which animals with parietal lesions had presurgical experience on spatial reversals (Weir \& Thomas, 1975). Rats with temporal lesions apparently have not been tested on spatial reversals. However, Killackey et al. (1971) reported that a single temporally operated tree shrew that was incapable of improving its performance on a black-white reversal learning-set task was capable of learning a spatial reversal task. Since Weir and Thomas (1975) had the complicating factor

Table 1

Diameters (in Millimeters) and Trephine Placements (in Millimeters) in Reference to Sagittal and Coronal Sutures for the Four Cortical Ablations

\begin{tabular}{lccc}
\hline \multicolumn{1}{c}{ Lesion } & Diameter & Sagittal & Coronal* \\
\hline Parietal & 3.5 & 1.0 & -1.0 \\
Temporal & 2.5 & 6.0 & -6.0 \\
Frontopolar & 3.5 & 1.0 & 1.0 \\
Striate & 5.5 & 1.0 & -7.5 \\
\hline
\end{tabular}

Note-All ablations were bilateral. $\quad{ }^{*}$ Negative numbers indicate placements caudal to this suture. The frontopolar ablations were rostral to this suture. of preoperative training and since rats with temporal lesions have not been tested on spatial reversals, it seemed useful to compare the effects of parietal and temporal lesions in the rat on the ability to acquire spatial reversals postoperatively. Such an experiment would further test the hypothesis that reversal learning deficits following temporal lesions are specific to the visual modality.

\section{Methods}

Subjects. Rats with parietal $(n=6)$, temporal $(n=6)$, or frontopolar $(n=6)$ lesions and nonoperated rats $(n=6)$ were tested. Training began 14 days postoperatively.

Apparatus and Procedure. The apparatus was a two-choice alley which consists of a startbox which opens into an area where the rat must choose between two parallel alleys. The startand goalboxes $(29 \times 14.5 \times 13.5 \mathrm{~cm})$ were constructed to be interchangeable. All compartments were divided by guillotine doors to prevent the animal from correcting incorrect choices. A food dish was located at the rear-center of each goal box. The distance of a run from the startbox to the choice point was $37.5 \mathrm{~cm}$ and from the choice point to the food dish was $25.5 \mathrm{~cm}$. Height and width dimensions of the alley were the same as the goal- and startboxes. Reward always consisted of two 45-mg Noyes food pellets.

Ten days prior to surgery, all animals were given free access 5 min daily to a food dish containing six Noyes food pellets in the home cage. Twelve days following surgery, the rats were food-deprived for $48 \mathrm{~h}$, and on Day 14 the rats were adapted to the apparatus by being placed in each of the goalboxes with both food dishes baited. On Days 15 and 16, the animals were placed in the startbox and allowed to explore the apparatus for $10 \mathrm{~min}$ each day, with rewards located in both goalboxes. Alley running was conditioned by giving the animals 4 days of training, 10 trials each day, in which responses to both goalboxes were reinforced. On Day 21, spatial reversal training began with reward available only in the goalbox which was in the position less frequently chosen during alley-running conditioning. Animals were given 10 noncorrection trials per day until a criterion of eight correct responses in one testing session was attained. On the next day, the previously incorrect alley became correct and remained so until criterion was again attained. Errors were signaled by the onset of a light $(50 \mathrm{~W})$ and a buzzer $(58 \mathrm{~dB}$; re .0002 dynes $/ \mathrm{cm}^{2}$; Weir \& Thomas, 1975) for $1 \mathrm{sec}$. The rats were trained on a total of seven reversals and were given 11 to $13 \mathrm{~g}$ of food following each daily session, with free access to water.

\section{Results}

A two-way analysis of variance with repeated measures on one factor (reversals) was computed on the errors data. One parietal animal was eliminated from the study when, on the second reversal, it had made more than three times the median number of errors for the rest of its group. The appropriate adjustments were made for unequal ns. The analysis indicated that only the lesion group difference was significant $[F(3,20)=7.22, \quad p<.01]$. Figure 1 suggests, and a Newman-Keuls analysis confirms, that parietal rats committed more errors than all other groups (ps $<.01)$.

An anatomical examination of the brains, recon- 


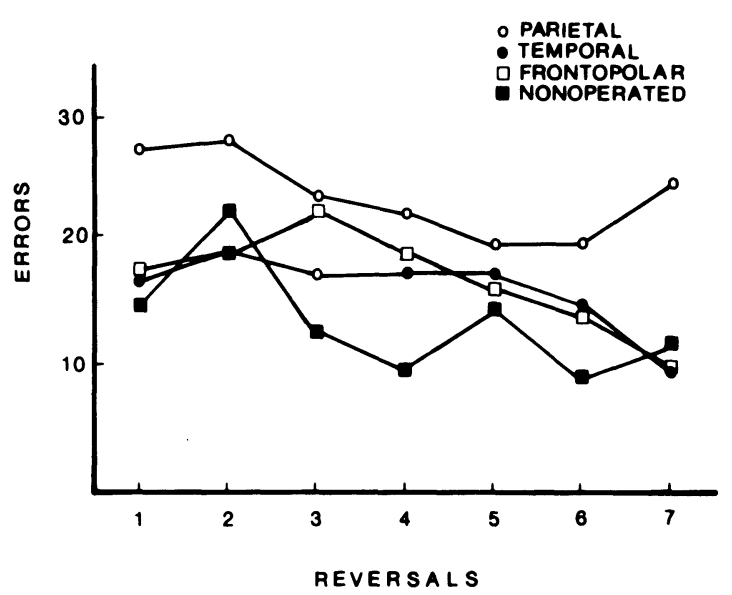

Figure 1. Error scores for each group on the spatial reversal task.

structed in Figure 2, indicated that the parietal and temporal lesions were generally within the intended cortical site. It should be mentioned that the parietal ablations seen here tended to be located primarily in the most rostral portions of the parietal cortex and sometimes included portions of somatosensory area I. The frontopolar lesions conformed to those studied by Thomas and Weir (1975). A Wilcoxon and Wilcox multiple comparisons analysis was performed on the ranked cortical lesion sizes, but statistically significant group differences were not seen.

Subcortical damage incidentally inflicted by the
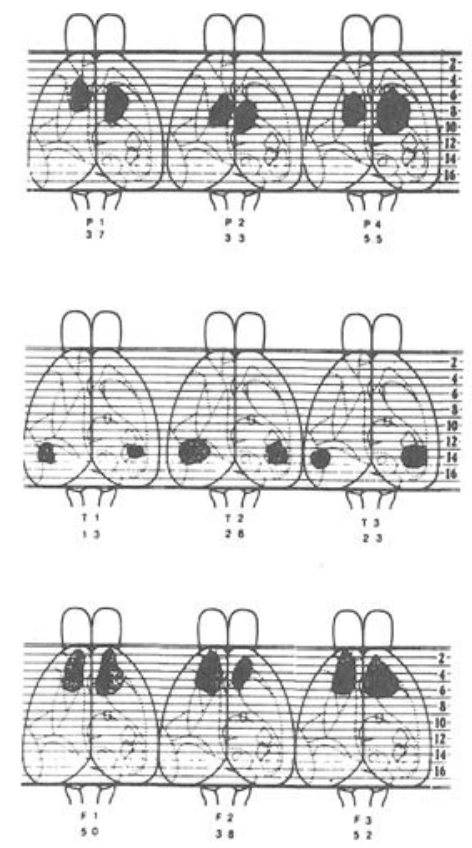

parietal lesions included the corpus callosum in three animals and a small portion of the hippocampus in one animal. Two animals with temporal lesions sustained damage to the corpus callosum, and one had slight damage to the hippocampus. One animal with a frontopolar lesion had slight damage to the corpus callosum. Thalamic gliosis and cell loss following parietal lesions was consistently associated with the lateral nucleus, pars principalis, and the posterior thalamic nucleus in two cases. Temporal ablations were associated with gliosis and cell loss in the lateroposterior nucleus and the medial portions of the lateral geniculate nucleus.

\section{EXPERIMENT 2}

While the integrity of the striate cortex has been shown to be important for pattern vison in the rat (Horel, Bettinger, Royce, \& Meyer, 1966; Lashley, 1931), Spear and Barbas (1975) have suggested that the deleterious effects of visual cortex lesions on pattern discriminations may not be absolute. These investigators have shown that lesions of the visual cortex placed in two stages with training between operations do not abolish the ability to make a discrimination between horizontal and vertical stripe stimuli, while removal of this region in one stage results in an operationally defined inability to learn the discrimination. In contrast to the rat, tree shrews with striate ablations performed in one stage are capable of discriminating upright from inverted
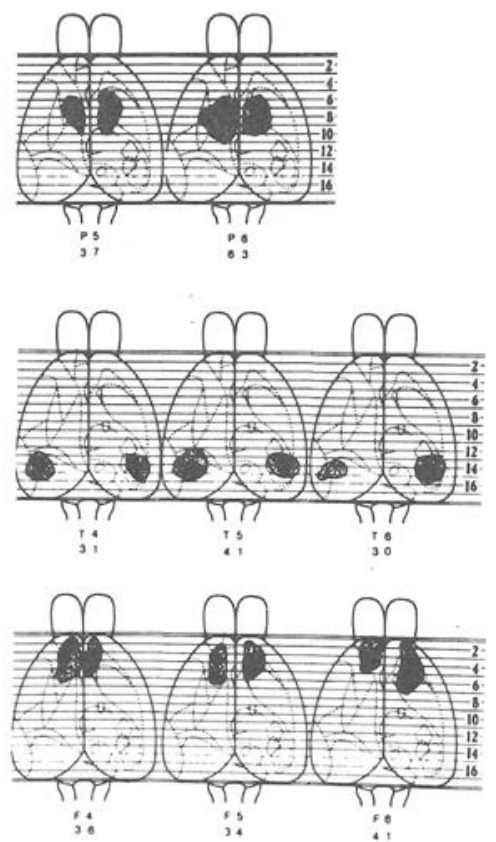

Figure 2. Reconstructions of the dorsal views of the cortical lesions examined in Experiment 1. Dark portions represent areas of deepest destruction for that animal. Stippled areas indicate areas of slight cortical damage. The percentage of total cortex destroyed is shown under each brain. 
triangles but are incapable of discriminating these stimuli when they are enclosed by annuli. The preservation of a second visual pathway from the tectum to the pulvinar, and hence to the extrastriate cortex, has been suggested as being responsible for the continued visual behavior of tree shrews after striate ablations (Diamond \& Hall, 1969; Killackey et al., 1971; Killackey, Wilson, \& Diamond, 1972; Snyder \& Diamond, 1966). Since temporal cortex ablations in the rat (Boyd \& Thomas, 1977) appear to produce deficits on a black-white reversal task similar to those seen in temporally ablated tree shrews (Killackey, Diamond, Hall, \& Hudgins, 1968; Killackey et al., 1971), it seemed useful to ask whether this visual function of the temporal cortex in the rat is dependent upon its thalamic projections from the lateroposterior nucleus.

\section{Methods}

Subjects. Rats with electrolytic lesions of the lateroposterior thalamic nucleus $(n=6)$, ablations of either the temporal association cortex $(n=6)$ or striate cortex $(n=6)$, and nonoperated controls $(n=6)$ were trained on this task, beginning 14 days postoperatively.

Apparatus and Procedure. Surgical procedures were similar to those described earlier, with coordinates for the lateroposterior lesions being $-3.2 \mathrm{~mm}$ posterior to suture coronalis, $2.75 \mathrm{~mm}$ lateral to suture sagittalis, and $5.25 \mathrm{~mm}$ below the surface of the brain. The apparatus and adaptation procedures described in Experiment 1 were used in this study. The discriminanda were constructed from black and white construction paper and were mounted behind Plexiglas panels which comprised the rear wall of the goalboxes $(14.5 \times 13.5 \mathrm{~cm})$. These stimuli were backilluminated (Lafayette 1650 visual stimulator), and photometric readings (SEI Photometer) were $.02 \mathrm{fL}\left(.07 \mathrm{~cd} / \mathrm{m}^{2}\right)$ for the black stimulus and $32 \mathrm{fL}\left(109.64 \mathrm{~cd} / \mathrm{m}^{2}\right)$ for the white stimulus. Room illumination was provided by a $25-\mathrm{W}$ bulb located at the bottom center of the table supporting the apparatus. The animals received 40 pretraining trials in which both food dishes were baited to condition alley running and to determine stimulus preference. On the next day, training began with only choices of the nonpreferred stimulus (determined by simple majority of pretraining responses) reinforced. The light-buzzer response to incorrect choices was the same as that employed in Experiment 1. Position of the incorrect discriminanda was determined by the Gellerman (1933) series. The training procedure was similar to that used by Boyd and Thomas (1977) and Snyder and Diamond (1968) and included the following. When an incorrect alley choice was made, the trial was scored as an error; however, the position of the stimuli remained the same until a correct response was made. Only then was the position of the stimuli changed and a new trial begun. The animals were given 10 trials per day until a criterion of either eight consecutive correct responses in a single session or 2 consecutive days of $80 \%$ correct responses were attained.

\section{Results}

A two-way analysis of variance with repeated measures on one factor (reversals) was performed on the error scores. One lateroposterior animal died early during training, and the appropriate adjustments were made for unequal ns.

The analysis of variance indicated that the Lesion
Group $[\mathrm{F}(3,20=3.49, \quad \mathrm{p}<.05]$ and Reversal $[F(5,100)=18.39, p<.01]$ effects were significant. A Newman-Keuls analysis of the Lesion Group effect indicated that the temporal group made more errors than did nonoperated controls $(p<.01)$, striate ablated animals $(\mathrm{p}<.05)$, and animals with lateroposterior lesions $(p<.05)$. Figure 3 presents the error scores for each group on each reversal. Newman-Keuls analysis of the significant Reversal effect showed that all groups tended to make more errors on Reversals 2 and 3 than on all other reversals (ps $<.01)$. Also, more errors were made on Reversal 4 than on Reversal 1 ( $p<.01)$.

Inspection of the dorsal surface of each brain shown in Figure 4 indicated that the lesions were within the intended cortical sites. It should be mentioned, however, that the striate lesions often exceeded the boundaries of area 17 and included portions of the extrastriate regions 18 and 18A (Krieg, 1946). A Mann-Whitney analysis of the ranked temporal and striate ablations indicated that the temporal ablations $(\mathrm{Mdn}=3.4 \%)$ were significantly smaller than the striate ablations $(\mathrm{Mdn}=6.9 \%)$ $[U=0.0, p<.001]$. This is of little concern, however, since the temporal group had the most errors and since there was no difference between the striate and nonoperated control animals on errors committed.

Incidental subcortical damage included the corpus callosum for three animals with temporal lesions and four animals with striate lesions and the superficial layer of the superior colliculus for one animal with a striate lesion. All lateroposterior lesions involved the intended nucleus and generally extended from A-P 3.0 to $2.0 \mathrm{~mm}$ and D-V 5.0 to $6.5 \mathrm{~mm}$. Other thalamic nuclei affected included the medial portion of the lateral geniculate and the posterior thalamic nucleus in all cases, the rostral medial geniculate in four animals, the ventral thalamic nucleus in three

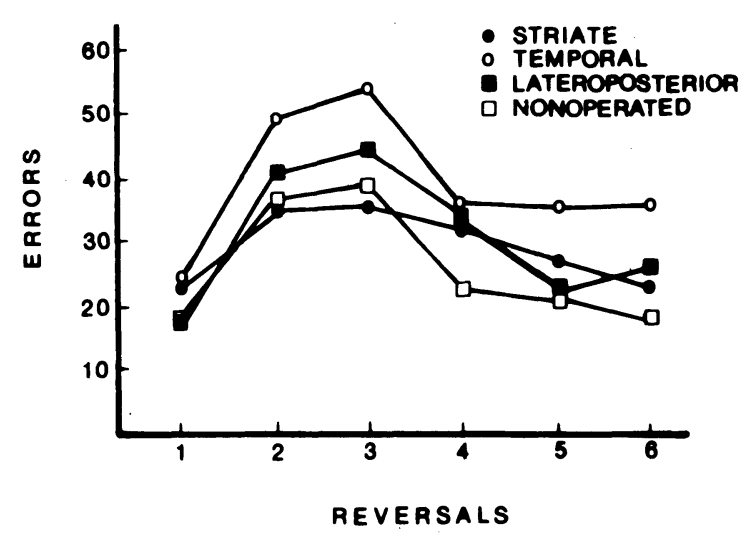

Figure 3. Error scores for each group on the black-white reversal task. 

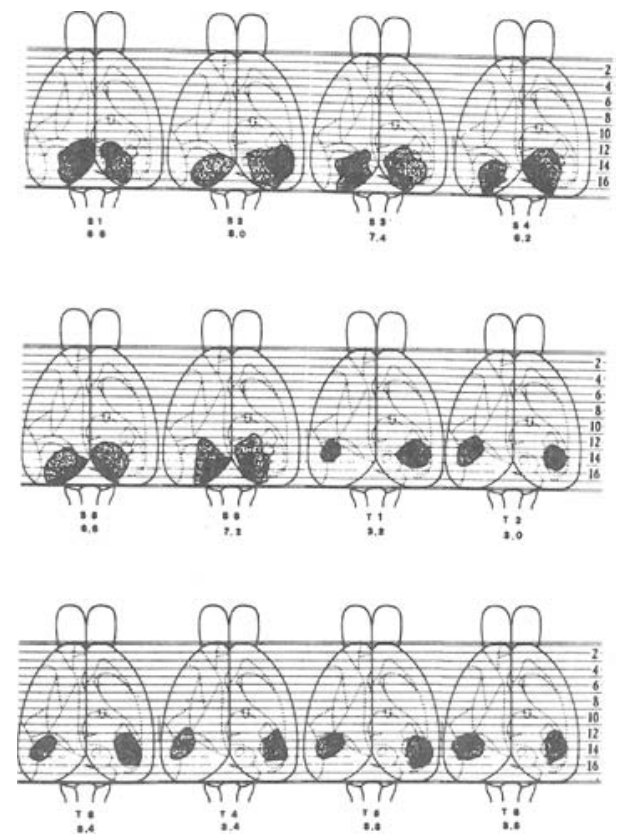

Figure 4. Reconstructions of the dorsal views of the cortical lesions examined in Experiment 2. The descriptions in Figure 2 apply here.

animals, the caudal lateral thalamic nucleus -(pars principalis) in three animals, and the rostral superior colliculus in four cases. A representative lateroposterior lesion is shown in Figure 5.

Thalamic gliosis was associated with the lateroposterior nucleus and the medial portions of the lateral geniculate following temporal ablations. Striate ablations produced gliosis located exclusively in the lateral geniculate nucleus.

\section{GENERAL DISCUSSION}

The results of these experiments, taken with previous research, indicate that there is a dissociation of the effects of parietal and temporal association cortex lesions. While parietal ablations were followed by impaired performance on a spatial reversal task, they apparently produced no deficit on the blackwhite reversal task in Boyd and Thomas' (1977) study. Rats with temporal ablations comprising a median of $2.8 \%$ total cortex were not impaired on a spatial reversal, but animals with temporal ablations comprising a median of $3.4 \%$ total cortex were impaired on a black-white reversal task. A Mann-Whitney analysis of the ranked percentage temporal damage between these two studies was nonsignificant $(U=7.50, p>.05)$. This suggests that the reversal deficit seen following temporal ablation in the rat is specific to the visual modality. It is interesting to note that the effects of parietal and temporal association cortex damage in advanced primates are similarly dissociated (Pribram \& Barry, 1956; Wilson, 1957). The findings reported here support Boyd and Thomas' (1977) suggestion that, in the interest of primate conservation, the rat may be a useful subject in preliminary investigations of posterior association cortex function.

The finding that parietal damage results in impaired spatial reversal performance strongly implicates the loss of the ability to utilize spatial cues as an explanation for the deficits seen on mazes and maze-like tasks (Ain et al., 1969; Boyd \& Thomas, 1977; Forgays, 1952; Gross et al., 1965; Krechevsky, 1935; Lansdell, 1953; Thomas, 1966; Thomas \& Weir, 1975). This interpretation requires qualification, since preoperative training on spatial reversals (Weir \& Thomas, 1975) appears to negate the deleterious effects of parietal lesions.

The lateroposterior thalamic lesions studied here did not result in the deficit seen on black-white reversals following temporal ablations. This evidence is consistent with a recent study reported by Hughes $\left(1977^{1}\right)$ in which rats with striate, lateroposterior, or combined striate and lateroposterior nucleus lesions were tested on the ability to relearn a blackwhite discrimination and a striped pattern discrimination. On the black-white discrimination, only the animals with combined lesions evinced a deficit. On the pattern discrimination, both the striate and combined striate and lateroposterior lesion groups demonstrated deficits; however, the rats with striate cortex damage were able to learn the stripe discrimination in fewer trials than rats with combined lesions. No deficit was seen in the group with lateroposterior nucleus destruction. Since the temporal cortex in the rat receives projections from both the striate cortex (Montero, Bravo, \& Fernandez, 1973) and the lateroposterior thalamic nucleus (Hughs, 1977; Lashley, 1941; McDaniel et al., 1978), it may be that

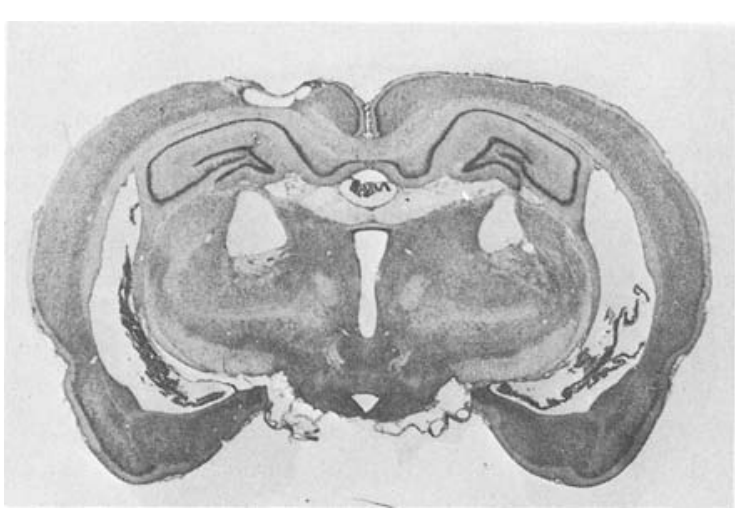

Figure 5. A representative lateroposterior lesion. 
the preservation of connections from either region is sufficient to enable the temporal cortex to function normally on the black-white reversal task. A test of this latter hypothesis would involve comparing animals with combined lesions in both the striate cortex and the lateroposterior nucleus with temporal animals on a black-white reversal task.

\section{REFERENCES}

Ain, B. R., Lubar, J. F., Moon, R. D., \& Kulig, R. M. Effect of septal and neocortical damage on complex maze learning. Physiology \& Behavior, 1969, 4, 235-238.

Boyd, M. G., \& Thomas, R. K. The effects of posterior association cortex lesions in rats: Mazes, pattern discrimination, and reversal learning. Physiological Psychology, 1977, 5, 455-461.

Diamond, I. T., \& Hall, W. C. Evolution of neocortex. Science, 1969, 164, 251-262.

Forgays, D. G. Reversible disturbances in rats following cortical insult. Journal of Comparative and Physiological Psychology, 1952, 45, 216-225.

Gellerman, L. W. Chance orders of alternating stimuli in visual discrimination experiments. Journal of Genetic Psychology, 1933, 42, 206-208.

Gross, C. G., Chorover, S. L., \& Cohen, S. M. Caudate, cortical, hippocampal and dorsal thalamic lesions in rats, alternation and Hebb-Williams maze performance. Neuropsychologia, 1965, 3, 53-68.

Harting, J. K., Hall, W. C., \& Diamond, I. T. Evolution of the pulvinar. Brain, Behavior and Evolution, 1972, 6, 424-452.

Horel, J., Bettinger, L., Royce, J., \& Meyer, D. R. Role of neocortex in the learning and relearning of the two visual habits by rats. Journal of Comparative and Physiological Psychology, 1966, 61, 66-78.

Hughs, H. C. Anatomical and neurobehavioral investigations concerning the thalamic-cortical organization of the rat's visual system. Journal of Comparative Neurology, 1977, 175, 311-336.

Killackey, H., Diamond, I. T., Hall, W. C., \& Hudgins, G. An ablation study of the tecto-pulvinar-extrastriate system in the squirrel and tree shrew. Federation Proceedings, 1968, 27, 517.

Killackey, H., Snyder, M., \& Diamond, I. T. Function of striate and temporal cortex in the tree shrew. Journal of Comparative and Physiological Psychology Monograph, 1971.

Killackey, H., Wilson, M., \& Diamond, I. T. Further studies of the striate and extrastriate visual cortex in the tree shrew. Journal of Comparative and Physiological Psychology, 1972, 81, 45-63.

KRECHEVSKY, I. Brain mechanisms and "hypotheses." Journal of Comparative Psychology, 1935, 19, 425-468.
KRIEG, W. J. S. Connections of the cerebral cortex. 1. The albino rat. A. Topography of the cortical areas. Journal of Comparative Neurology, 1946, 84, 221-275.

LANSDELL, H. C. Effects of brain damage on intelligence in rats. Journal of Comparative and Physiological Psychology, 1953, 46, 461-464.

LASHLEY, K. S. The mechanisms of vision IV. The cerebral areas necessary for pattern vision in the rat. Journal of Comparative Neurology, 1931, 53, 419-478.

LASHLEY, K. S. Thalamo-cortical connections of the rat's brain. Journal of Comparative Neurology, 1941, 75, 67-121.

McDaniel, W. F., McDaniel, S. E., \& Thomas, R. K. Thalamocortical projections to the temporal and parietal association cortices in the rat. Neuroscience Letters, 1978, 7, 121-125.

Montero, V. M., Bravo, H., \& Fernandez, V. Striateperistriate cortico-cortical connections in the albino and grey rat. Brain Research, 1973, 53, 202-207.

Pribram, H. B., \& BARRY, J. Further behavioral analysis of parieto-temporo-preoccipital cortex. Journal of Neurophysiology, 1956, 19, 99-106.

SNyder, M., \& Diamond, I. T. The organization and function of the visual cortex in the tree shrew. Brain, Behavior and Evolution, 1968, 1, 244-288.

SNYDER, M., Hall, W. C., \& Diamond, I. T. Vision in tree shrew (Tupia glis) after removal of striate cortex. Psychonomic Science, 1966, 3, 243-244.

Spear, P. D., \& Barbas, H. Recovery of pattern discrimination ability in rats receiving serial or one-stage visual cortex lesions. Brain Research, 1975, 94, 337-346.

Thомаs, R. K., JR. Immediate and subsequent effects of brain damage in rats. Journal of Comparative and Physiological Psychology, 1966, 62, 472-475.

Thomas, R. K., JR., \& Peacock, L. J. A method of measuring brain lesions. Psychonomic Science, 1965, 3, 184.

Thomas, R. K., \& WeIR, V. K. The effects of lesions in the frontal or posterior association cortex of rats on Maze III. Physiological Psychology, 1975, 3, 210-214.

WEIR, V. K., \& THомAS, R. K. Failure to find spatial reversal deficits following medial frontal lesions. Bulletin of the Psychonomic Society, 1975, 5, 465-468.

Wilson, M. Effects of circumscribed cortical lesions upon somesthetic and visual discrimination in the monkey. Journal of Comparative and Physiological Psychology, 1957, 50, 630-635.

\section{NOTE}

1. The Hughs article (1977) was published after the experiments discussed here had been performed.

(Received for publication January 25, 1978; revision accepted June 14,1978 .) 